\title{
Gene editing in bacteria via SpCas9/sgRNA ribonucleoprotein complexes
}

\author{
Arroyo-Olarte Rubén Dario ${ }^{1}$, Bravo Rodríguez Ricardo ${ }^{1}$, Morales-Ríos Edgar ${ }^{1 *}$ \\ ${ }^{1}$ Departamento de Bioquímica, Centro de Investigación y Estudios Avanzados, Instituto \\ Politécnico Nacional, Ciudad de México, México \\ *Corresponding author: edgar.morales@cinvestav.mx
}

\begin{abstract}
Gene editing has been revolutionized by CRISPR (Clustered Regularly Interspaced Short Palindromic Repeats)-Cas technology in a variety of organisms. In bacteria, however, CRISPR-Cas still holds many caveats such as high toxicity of Cas9 and off-target editing effects. In this work we develop a system for the incorporation of Cas9/single guide RNA ribonucleoprotein complexes in bacteria and their successful application for gene editing via homologous recombination. Targeting of a green fluorescent protein (GFP) reporter allows for easy verification of gene-edition via conversion to blue-fluorescent protein (BFP), mediated by the well characterized 196T > C (Tyr66His) mutation.
\end{abstract}

Key words: CRISPR-Cas, ribonucleoprotein, gene-editing, GFP, BFP.

\section{Introduction}

Feasibility of the CRISPR-Cas system among prokaryotes varies greatly depending on several factors, e.g. Cas proteins cytotoxicity, AT genome content and available genetic transformation methods. In this regard, evaluation of loss of fluorescence in GFP-expressing bacteria serves as a straightforward way to assess CRISPR-Cas activity in vivo in different species. This has been applied in E. coli with a 2-plasmid system, one encoding for Cas9 and GFP-specific sgRNA expression, and another for GFP expression. GFP-plasmid loss varied between $80 \%$ to $98 \%$ of colonies depending on the sgRNA sequence [1]. The system can also be used to assess the efficiency of CRISPR-Cas-mediated gene editing. It has been shown that the Tyr66-His mutant (encoded by the single base substitution 196T $>$ C) shifts wild-type GFP absorption and emission towards the blue spectrum, thus creating blue 
fluorescent protein (BFP) (Fig. 1) [2]. The loss of the hydrogen-bond provided by tyrosin side-chain hydroxyl group, also generates a slightly more relaxed tertiary structure in BFP (Fig. 1). This amino acid change is involved as well in the higher stability of BFP fluorescence at acidic $\mathrm{pH}$, but lower at alkaline $\mathrm{pH}$ compared to GFP [3].

A GFP to BFP conversion assay has recently been applied to evaluate a plasmid-based CRISPR/Cas9 system in Methylococcus capsulatus [4]. Transformation with Cas9/single guide (sg)RNA ribonucleoprotein (RNP) complexes carries several advantages. The main advantage of this approach is that it does not rely on the host transcription and translation machinery. Besides, the RNP complex is usually degraded shortly after transfection, avoiding the toxic effects of a continuous Cas9 expression (Fig 4). It also does not require cloning. Therefore, there is no restriction in the selection of sgRNAs that may target a cloning strain genome. It also presents a more concise streamline than the plasmid methods, as no plasmid curing is required. In this work we applied the RNP approach in a GFP to BFP conversion assay [5] and show successful gene editing in Escherichia coli, and potentially in other prokaryotic organisms, by homologous recombination with an exogenous DNA repair template.

\section{Materials}

\subsection{Plasmids, PCR, sgRNA in vitro transcription and nuclease assays}

1. pET-NLS-Cas9-6xhis and pAKgfp1 are requested from Addgene (https://www.addgene.org), with ID no. 62934 and 14076, respectively. The pETNLS-Cas9-6xhis plasmid is used to express recombinant $S p$ Cas9 for affinity purification, while pAKgfp1 plasmid is used to express GFP in Escherichia coli.

2. DNA oligonucleotides are requested to be used as homology-directed repair (HDR) template, for DNA template synthesis by overhang PCR for sgRNA in vitro transcription (IVT), and for Cas9 substrate PCR to be used in nuclease assays (Table 1): 
Table 1. Oligonucleotides used in this study

\begin{tabular}{lll} 
Name & Sequence & Purpose \\
\hline GFPtoBFP-RT & $\begin{array}{l}\text { ACTGGAAAACTACCTGTTCCATGGCCAACACTTGTCACTACTCTG } \\
\text { AGCCACGGTGTTCAATGCTTTGGAGATACCCAGATCATATGAAA template }\end{array}$ & $\begin{array}{l}\text { HDRP } \rightarrow \text { BFP) } \\
\text { (GFP }\end{array}$ \\
\hline Sp-GFPmut178-F & $\begin{array}{l}\text { GGAGGCCGGAGAATTGTAATACGACTCACTATAGGGCTTG } \\
\text { TCACTACTTTCGGGTATTTAGAGCTAGAAATAGCAAG }\end{array}$ & \\
Sp-GFPmut178-F2 & $\begin{array}{l}\text { GGAGGCCGGAGAATTGTAATACGACTCACTATAGGGCTTG } \\
\text { TCACTACTTTCGGTTAGTTTAGAGCTAGAAATAGCAAG }\end{array}$ & $\begin{array}{l}\text { DNA template } \\
\text { for sgRNA IVT }\end{array}$ \\
SpCas9-scaff-R & AAAAAAGCACCGACTCGGTGCCACTTTTTCAAGTTGATAAC & \\
\hline GFPmut3-F & ATACTAGCCTTATTTAACTTGCTATTTCTAGCTCTAAAAC & \\
GFPmut3-R & TTTGTATAGTTCATCCATGCCATG & $\begin{array}{l}\text { Cas9 substrate } \\
\text { for nuclease } \\
\text { assay }\end{array}$ \\
\hline
\end{tabular}

3. TranscriptAid T7 high yield transcription kit (Thermo Fischer Scientific).

4. Common molecular biology reagents, e.g., nuclease-free water, ethyl alcohol, isopropyl alcohol, agarose, proteinase K, RNAseZAPTM (Sigma-Aldrich), Taq DNA polymerase, DNA purification spin-column system, ethidium bromide/SYBR green.

5. 10x Cas9 reaction buffer: $100 \mathrm{mM} \mathrm{NaCl}, 50 \mathrm{mM}$ Tris- $\mathrm{HCl}, 10 \mathrm{mM} \mathrm{MgCl}, 100 \mu \mathrm{g} / \mathrm{ml}$ BSA, pH 7.9.

\subsection{Escherichia coli strains and culture}

1. Competent bacterial cells: Escherichia coli strain soluBL21 ${ }^{\mathrm{TM}}$ (Genlantis) for recombinant SpCas9-6xhis expression and purification, and E. coli strain TOP10 ${ }^{\mathrm{TM}}$ (Thermo Fischer) for GFP expression and gene editing.

2. Luria-Bertani agar plates supplemented with ampicillin (Sigma-Aldrich).

3. 2 xYT medium: $16 \mathrm{~g} / \mathrm{L}$ tryptone, $10 \mathrm{~g} / \mathrm{L}$ yeast extract, $5.0 \mathrm{~g} / \mathrm{L} \mathrm{NaCl}$.

4. Isopropyl $\beta$-galactoside (IPTG, Sigma-Aldrich).

5. Swinging bucket centrifuge and microcentrifuge.

6. Temperature-controlled shaker and thermoblock.

\subsection{Escherichia coli electroporation}

1. $0.1 \mathrm{~cm}$-gap sterile electroporation cuvettes (Biorad).

2. Gene Pulser X-Cell electroporation system (Biorad).

3. Phosphate-buffered saline (PBS) supplemented with $10 \%$ glycerol.

4. SOC medium: $0.5 \%(\mathrm{w} / \mathrm{v})$ yeast extract, $2 \%(\mathrm{w} / \mathrm{v})$ tryptone, $10 \mathrm{mM} \mathrm{NaCl}, 2.5 \mathrm{mM}$ $\mathrm{KCl}, 20 \mathrm{mM} \mathrm{MgSO}_{4}, 20 \mathrm{mM}$ glucose. 


\subsection{Recombinant SpCas9-6xhis purification}

1. Ultrasonic homogenizer VC-500 (Cole-Palmer).

2. ÄKTA pure 25L purification system (Cytiva).

3. $5 \mathrm{ml}$ Nickel-nitrilotriacetic acid (Ni-NTA) HisTrapTM column (Cytiva).

4. Gel filtration Superdex 200 increase column (Cytiva).

5. Amicon $100 \mathrm{KDa}$ centrifugal filter units (Sigma-Aldrich).

6. Lysis buffer 10x: $300 \mathrm{mM}$ Tris, $2 \mathrm{M} \mathrm{NaCl}, 20 \mathrm{mM} \mathrm{MgCl}_{2}, \mathrm{pH}$ 7.5.

7. Buffer A: lysis buffer $1 \mathrm{x}, 5 \mathrm{mM}$ imidazole, $2 \mathrm{mM}$ benzamidine, $0.8 \mathrm{mM}$ phenylmethylsulfonyl fluoride (PMSF), 3 mM $\beta$-mercaptoethanol, $\mathrm{pH}$ 7.0.

8. Buffer B: lysis buffer $1 \mathrm{x}, 500 \mathrm{mM}$ imidazole, $2 \mathrm{mM}$ benzamidine, $0.8 \mathrm{mM}$ PMSF, 3 $\mathrm{mM}, \beta$-mercaptoethanol, $\mathrm{pH}$ 7.0.

9. Sodium dodecyl sulphate (SDS), polyacrylamide, electrophoresis chamber.

10. Cas9 storage buffer: $50 \mathrm{mM}$ 4-(2-hydroxyethyl)-1-piperazineethanesulfonic acid (HEPES), $300 \mathrm{mM} \mathrm{NaCl}, 0.5 \mathrm{mM} \beta$-mercaptoethanol, 10\% glycerol, $2 \mathrm{mM}$ benzamidine, $0.8 \mathrm{mM}$ PMSF.

11. Coomassie Brilliant Blue G-250 for Bradford protein assay.

12. UV-vis spectrophotometer and $1 \mathrm{~cm}$-gap cuvettes.

\section{Methods}

\subsection{Cell culture and SpCas9-6xhis expression}

Escherichia coli strain soluBL21 ${ }^{\mathrm{TM}}$ (Genlantis) was transformed by heat-shock (40-45 sec, $42{ }^{\circ} \mathrm{C}$ ) with plasmid pET-NLS-Cas9-6xhis (Addgene). Transformants were selected by culturing in Luria-Bertani agar plates supplemented with $150 \mu \mathrm{g} / \mathrm{ml}$ ampicillin (SigmaAldrich). Recombinant SpCas9-6xHis expression was induced by culturing in 2xYT medium (16 g/L tryptone, $10 \mathrm{~g} / \mathrm{L}$ yeast extract, $5.0 \mathrm{~g} / \mathrm{L} \mathrm{NaCl}$ ) supplemented with $1 \mathrm{mM}$ isopropyl $\beta$ galactoside (IPTG, Sigma-Aldrich), for $16-18 \mathrm{~h}$ at $18{ }^{\circ} \mathrm{C}$. Cells were centrifuged $(4,000 \mathrm{xg}$, $30 \mathrm{~min}$ ), and pellets were stored at $-70{ }^{\circ} \mathrm{C}$.

\subsection{Ni-NTA affinity and size-exclusion chromatography}

Cell pellets were lysed by sonication (50\%, $10 \mathrm{~min}: 30 \mathrm{~s}$ pulse, $5 \mathrm{~s}$ rest). Lysate was centrifuged $(30,000 \mathrm{xg}, 30 \mathrm{~min})$ to remove cell debris. Cleared lysate was filtered $(0.2 \mu \mathrm{m}$ Milipore) and affinity chromatography was performed on an ÄKTA pure 25L purification 
system (Cytiva). Sample was applied onto a $5 \mathrm{ml} \mathrm{Ni-NTA} \mathrm{HisTrap}{ }^{\mathrm{TM}}$ column (Cytiva) at a constant flow rate of $1.5 \mathrm{ml} / \mathrm{min}$. Next, unbound proteins were washed out at a flow rate of $3 \mathrm{ml} / \mathrm{min}$. A gradient solution containing imidazole from $5 \mathrm{mM}$ to $500 \mathrm{mM}$ was then applied to the column to elute bound proteins into $5 \mathrm{ml}$ fractions. Real-time UV spectrophotometrically $(260 \mathrm{~nm})$ allowed to visualize the elution of different protein peaks.

Eluted affinity fractions were separated by SDS-PAGE and gels were stained with Comassie blue. Fractions showing at least $80 \%$ purity of the expected SpCas9 protein band size (160 $\mathrm{kDa})$ were merged and concentrated by centrifugation $(3,200 \mathrm{xg})$ on a $100 \mathrm{kDa}$ Amicon filter unit.

Size-exclusion chromatography was then performed on a gel filtration Superdex 200 column (Cytiva) to further purify SpCas9 and to remove small molecule contaminants from elution buffer (e.g., imidazole). Final SpCas9-6xHis was re-concentrated on a $100 \mathrm{kDa}$ Amicon filter unit and dissolved in Cas 9 storage buffer. Final protein concentration was determined by a Bradford protein assay on a linear range of BSA standards $(0-20 \mu \mathrm{g} / \mathrm{ml})$.

\subsection{In vitro transcription of $\operatorname{sgRNAs}$}

First, the DNA template was generated by overhang PCR by mixing 1-unit Taq polymerase, $30 \mu 1$ sgRNA-specific forward primer (10 $\mu \mathrm{M}$ Sp-GFPmut178-F/F2), $20 \mu 1$ of reverse primer (10 $\mu \mathrm{M}$ SpCas9-scaff-R) and 1x reaction buffer. Forward and reverse oligonucleotides share a 25-bp overlapping sequence. The amplification protocol used will depend on the selected DNA polymerase, but a good approximation is as follows:

i) $\quad 94{ }^{\circ} \mathrm{C}$ for $2: 00$ minutes

ii) $\quad 94{ }^{\circ} \mathrm{C}$ for 30 seconds

iii) $\quad 55-58{ }^{\circ} \mathrm{C}$ for 30 seconds

iv) $\quad 68-72{ }^{\circ} \mathrm{C}$ for 25 seconds

v) Cycle steps ii to iv for 34 times

vi) $\quad 68-72{ }^{\circ} \mathrm{C}$ for 5 minutes 
PCR product is cleaned up using a commercial spin-column based system and purified DNA template is then used for IVT according to manufacturer's instructions. Briefly, 500-2000 ng DNA template are mixed with reaction buffer, $2 \mu 1$ enzyme mix, $2 \mu 1$ ATP, $2 \mu 1$ CTP, $2 \mu 1$ GTP and $2 \mu \mathrm{UTP}\left(75 \mu \mathrm{M}\right.$ each). Reaction is run at $37^{\circ} \mathrm{C}$ for $5 \mathrm{~h}$, and sgRNA is purified by ethanol precipitation. sgRNA purity is assessed by $\mathrm{A}_{260} / \mathrm{A}_{280}$ ratio $(>1.8)$ and integrity is verified by agarose electrophoresis and UV visualization.

\subsection{In vitro nuclease assay}

The first step is the preparation of Cas9/gRNA RNP, by incubating the following mix at 25 ${ }^{\circ} \mathrm{C}$ for $15 \mathrm{~min}$ :

$1 \mu \mathrm{M}$ sgRNA

$+1 \mu \mathrm{M}$ recombinant SpCas9-6xhis

$5 \mu 1$ 10x Cas9 reaction buffer

$\mathrm{X}$ ul nuclease-free water

$40-45 \mu 1$

Thereafter 5-10 $\mu$ l Cas9 substrate (GFPmut3 PCR product, $300 \mathrm{nM}$ ) are added, and reaction is incubated at $37^{\circ} \mathrm{C}$ for $1 \mathrm{~h}$. Reaction is then stopped by adding $2 \mu \mathrm{l}$ proteinase $\mathrm{K}(10 \mathrm{mg} / \mathrm{ml})$ at room temperature for $20 \mathrm{~min}$. Reaction products are then analyzed by agarose gel electrophoresis. Densitometric analysis of uncut vs. cut DNA bands observed under UV is performed with Image J software (NIH) to evaluate the reaction efficiency.

\subsection{RNP electroporation}

First the following reagents are mixed:

10-20 $\mu \mathrm{g}$ sgRNA

+ 20-40 $\mu \mathrm{g}$ recombinant SpCas9-6xhis

$5 \mu 1$ Cas9 reaction buffer

$\mathrm{X}$ ul nuclease-free water

$50 \mu 1$ final volume

An initial incubation of $15 \mathrm{~min}$ at room temperature is performed to allow Cas9/gRNA RNP formation. Thereafter, 80-100 $\mu \mathrm{g}$ DNA repair oligonucleotide (GFP to BFP) are added and combined to $50 \mu \mathrm{l}$ competent GFP-expressing E. coli TOP10 (in PBS/glycerol $10 \%, 4{ }^{\circ} \mathrm{C}$ ). 
The cell suspension $(100-120 \mu \mathrm{l})$ is then transferred to $0.1-\mathrm{cm}$ cuvettes and electroporated in a Gene-PulserX (Bio-Rad) with the following parameters: 2 pulses, voltage $1.25 \mathrm{kV}$, capacitance $200 \mu \mathrm{F}$. $900 \mu \mathrm{l}$ SOC medium are immediately added and transformed cells are allowed to recover for $2 \mathrm{~h}, 37^{\circ} \mathrm{C}, 1,000 \mathrm{xg}$ before plating in LB-agar plates supplemented with ampicillin $(150 \mu \mathrm{g} / \mathrm{ml})$.

\section{Results}

\subsection{Purification of recombinant SpCas9}

Protein samples at every stage of purification were collected and analyzed using SDS-PAGE (Fig. 2A-B). During the first step, Ni-NTA affinity chromatography, an elongated peak to the right was observed containing SpCas9 (Fig. 2A). Most contaminant proteins appeared in the fractions with less imidazole. As imidazole gradient increased, fractions started to show a $160 \mathrm{kDa}$ band corresponding to SpCas9 as the dominant protein. During the second step, size-exclusion chromatography, there were three major peaks with elution volumes of 8.8 , 13.6 and $16.3 \mathrm{ml}$ (Fig. 2B). The first peak $(8.8 \mathrm{ml})$ showed several bands including a 160 $\mathrm{kDa}$ protein in SDS-PAGE. However, this elution volume was too low for the expected molecular size of monomeric SpCas9 on the Superdex 200 increase 10/300 GL column (Figure S1) and was assumed to be comprised of soluble aggregates. Fractions corresponding to the second peak $(13.6 \mathrm{ml})$ showed almost exclusively a $160 \mathrm{kDa}$ band which also corresponds to the expected elution volume of similar-size proteins globular proteins purified by the same gel-filtration column (Table S1). The third peak $(16.3 \mathrm{ml})$ showed a mixture of contaminant proteins of lower molecular size (Fig. 2B). There was also a fourth minor peak eluting at $20.6 \mathrm{ml}$ that matched a conductance peak corresponding to the desalting of lowmolecular weight imidazole (68 Da) carried on from the Ni-NTA affinity chromatography step. Finally, purified SpCas9-6xhis was concentrated to $3-5 \mu \mathrm{g} / \mu \mathrm{l}$ for further experiments.

\subsection{In vitro nuclease assay shows high and specific activity of recombinant SpCas9}

In order to analyze the efficacy of the purified SpCas9-6xHis as a potential gene-editing tool, nuclease assays were performed in combination or absence of a specific sgRNA. Our results show that our preparation has no background nuclease activity in absence of a sgRNA targeting the fluorochrome site of the GFP gene as DNA substrate (PCR product) (Fig.3). 
However, when incubating the DNA substrate with RNP complexes of purified SpCas96xHis plus either a perfect-match or $1 \mathrm{bp}$-mismatch (as close as $3 \mathrm{bp}$ from PAM) sgRNA, there was no difference in the efficiency of formation of a specific double strand break (96.2 \% vs $95.4 \%$ ) (Fig. 3).

\subsection{Electroporation of RNPs and a DNA repair template induces GFP to BFP conversion}

Transformation with the RNPs of a highly active Cas9 and sgRNA (Fig. 2C) by electroporation leads to colonies loss of plasmid-encoded GFP fluorescence in E. coli colonies (Fig. 4). Remarkably, when a short DNA recombination template (30 bp homology arms) encoding mutations responsible for the GFP to BFP transition (Tyr66 $\rightarrow$ His) is also used, we could observe blue-fluorescent cells in RNP-transfected cultures (Fig. 5). Although the observed efficiency is low $(<5 \%)$, these results are a proof of principle for the use of RNPs in bacteria, which to our knowledge has not been reported so far in these organisms. As the transformation efficiency of a SpCas9/gRNA complex would expectedly be limited due to its massive size, recombineering efficiency must be maximized. However, CRISPR nuclease cytotoxicity would be minimized to achieve editing.

\section{Discussion}

Since its discovery and application as a genome-modifying tool almost a decade ago [6], CRISPR-Cas has quickly become the standard gene-editing technology in eukaryotic and prokaryotic organisms. However, the versatility of CRISPR-Cas based tools in bacteria has not been as expanded as in eukaryotic organisms. One of these applications is the use of ribonucleoprotein sgRNA/Cas9 complexes and synthetic DNA oligonucleotide donors. The first requirement for the RNP method to work properly is a recombinant SpCas9 enzyme with precise and efficient DNA targeting and nuclease activity. Our 2-step purification protocol generated highly active SpCas9-6xHis from E. coli with a high enzymatic activity $(95 \%)$ to make a targeted double strand on a DNA substrate (Fig. 3). This result is superior to what others have reported for recombinant SpCas9 when purified by affinity chromatography alone [7], but equivalent to more laborious reports with three purification steps (IMAC, ion exchange and size exclusion chromatography) [8]. Our purification 
approach, therefore, enhances Cas9 specific nuclease activity without sacrificing protein yield. Moreover, the nuclease activity of our preparation was completely sgRNA dependent as SpCas9 alone did not trigger any cut on the DNA substrate (GFP gene).

In order to improve the observed low efficiency of in vivo gene-editing of Cas9/gRNA RNPs, despite a very high specific in vitro activity, several actions can be taken. Employing smaller Cas9 orthologs has been shown to radically improve the permeability of RNPs via electroporation [9]. Using bacterial strains expressing heterologous DNA recombination systems, e.g. Lambda Red, could also improve the efficiency of targeted gene-editing by the RNP method as has been shown for plasmid-based methods [10-12]. Another important aspect that can be improved is the RNP incorporation efficiency, especially in hard to electroporate cells such as thick-walled gram-positive bacteria. In this regard several approaches have been recently developed. These include the usage of cationic polymerderivatized Cas9 [13] and lipid-Cas9 protein/mRNA nanoparticles [14,15].

The main advantage of the RNP approach is that it does not rely on the bacterial strain transcription and translation machinery [16], allowing to directly pre-evaluate the efficacy of the RNP preparation by in vitro nuclease assays as shown in the present work. Additionally, the RNP complex is usually degraded shortly after transfection, avoiding the toxic effects of a continuous Cas9 expression that has been described in several bacteria [17].

\section{Acknowledgements}

We thank Enrique Alcaraz for technical assistance.

\section{Funding}

EMR grants: SEP-PRODEP: CINVESTAV-PTC-002, "Ruben Lisker" Biomedicine grant 2017, CONACYT CB2017-2018: A1-S-10743, SEP-CINVESTAV 2018: 1.

RDAO: CONACYT Postdoctoral Scholarship: Application No. 2019-000006-01NACV01211.

\section{References}

[1] J.N. Pieczynski, A. Deets, A. McDuffee, H. Lynn Kee, An undergraduate laboratory 
experience using CRISPR-cas9 technology to deactivate green fluorescent protein expression in Escherichia coli, Biochem. Mol. Biol. Educ. 47 (2019) 145-155. https://doi.org/10.1002/bmb.21206.

[2] R. Heim, D.C. Prasher, R.Y. Tsien, Wavelength mutations and posttranslational autoxidation of green fluorescent protein., Proc. Natl. Acad. Sci. 91 (1994) 1250112504. https://doi.org/10.1073/pnas.91.26.12501.

[3] I.A. Saeed, S.S. Ashraf, Denaturation studies reveal significant differences between GFP and blue fluorescent protein, Int. J. Biol. Macromol. 45 (2009) 236-241. https://doi.org/10.1016/j.ijbiomac.2009.05.010.

[4] T. Tapscott, M.T. Guarnieri, C.A. Henard, Development of a CRISPR/Cas9 system for Methylococcus capsulatus in vivo gene editing, Appl. Environ. Microbiol. 85 (2019) e00340-19. https://doi.org/10.1128/AEM.00340-19.

[5] A. Glaser, B. McColl, J. Vadolas, GFP to BFP Conversion: A Versatile Assay for the Quantification of CRISPR/Cas9-mediated Genome Editing., Mol. Ther. Nucleic Acids. 5 (2016) e334. https://doi.org/10.1038/mtna.2016.48.

[6] M. Jinek, K. Chylinski, I. Fonfara, M. Hauer, J.A. Doudna, E. Charpentier, A programmable dual-RNA-guided DNA endonuclease in adaptive bacterial immunity, Science (80-. ). 337 (2012) 816-821. https://doi.org/10.1126/science.1225829.

[7] M. Mehravar, A. Shirazi, M.M. Mehrazar, M. Nazari, In vitro pre-validation of gene editing by CRISPR/Cas9 ribonucleoprotein, Avicenna J. Med. Biotechnol. 11 (2019) 259-263.

[8] C. Anders, M. Jinek, In vitro enzymology of Cas9, in: Methods Enzymol., 2014: pp. 1-20. https://doi.org/10.1016/B978-0-12-801185-0.00001-5.

[9] L.C.S. Medeiros, L. South, D. Peng, J.M. Bustamante, W. Wang, M. Bunkofske, N. Perumal, F. Sanchez-Valdez, R.L. Tarleton, Rapid, selection-free, high-efficiency genome editing in protozoan parasites using CRISPR-cas9 ribonucleoproteins, MBio. 8 (2017) e01788-17. https://doi.org/10.1128/mBio.01788-17.

[10] T. Guo, Y. Xin, Y. Zhang, X. Gu, J. Kong, A rapid and versatile tool for genomic engineering in Lactococcus lactis, Microb. Cell Fact. 18 (2019) 22. https://doi.org/10.1186/s12934-019-1075-3.

[11] C.R. Reisch, K.L.J. Prather, The no-SCAR (Scarless Cas9 Assisted Recombineering) system for genome editing in Escherichia coli, Sci. Rep. 5 (2015) 15096. https://doi.org/10.1038/srep15096.

[12] W. Chen, Y. Zhang, Y. Zhang, Y. Pi, T. Gu, L. Song, Y. Wang, Q. Ji, CRISPR/Cas9-based Genome Editing in Pseudomonas aeruginosa and Cytidine Deaminase-Mediated Base Editing in Pseudomonas Species, IScience. 6 (2018) 222231. https://doi.org/10.1016/j.isci.2018.07.024.

[13] Y.K. Kang, K. Kwon, J.S. Ryu, H.N. Lee, C. Park, H.J. Chung, Nonviral Genome 
Editing Based on a Polymer-Derivatized CRISPR Nanocomplex for Targeting Bacterial Pathogens and Antibiotic Resistance, Bioconjug. Chem. 28 (2017) 957967. https://doi.org/10.1021/acs.bioconjchem.6b00676.

[14] L. Zhang, P. Wang, Q. Feng, N. Wang, Z. Chen, Y. Huang, W. Zheng, X. Jiang, Lipid nanoparticle-mediated efficient delivery of CRISPR/Cas9 for tumor therapy, NPG Asia Mater. 9 (2017) e441. https://doi.org/10.1038/am.2017.185.

[15] Q. Cheng, T. Wei, L. Farbiak, L.T. Johnson, S.A. Dilliard, D.J. Siegwart, Selective organ targeting (SORT) nanoparticles for tissue-specific mRNA delivery and CRISPR-Cas gene editing, Nat. Nanotechnol. 15 (2020) 313-320. https://doi.org/10.1038/s41565-020-0669-6.

[16] M.A. DeWitt, J.E. Corn, D. Carroll, Genome editing via delivery of Cas9 ribonucleoprotein, Methods. 121-122 (2017) 9-15. https://doi.org/10.1016/j.ymeth.2017.04.003.

[17] R.D. Arroyo-Olarte, R. Bravo Rodríguez, E. Morales-Ríos, Genome editing in bacteria: Crispr-cas and beyond, Microorganisms. 9 (2021) 844.

https://doi.org/10.3390/microorganisms9040844. 


\section{Figure legends}

Figure 1. Converting the green fluorescent protein to the blue fluorescent protein. Crystallographic structures of the GFB and BFP shows the amino acid residues close to the tyrosine 66 in GFP and the histidine 66 in BFP. The Tyr66-His mutation (encoded by a single nucleotide substitution at 196T $>$ C) has been shown to alter wild-type GFP uptake and blue spectrum luminescence, resulting in the blue fluorescent protein (BFP).

Figure 2. Purification of the recombinant Cas9 from Streptococcus pyogenes. During the first step, Ni-NTA affinity chromatography, an elongated peak to the right was observed containing SpCas9 (A) As the imidazole gradient increased, a band of $160 \mathrm{kDa}$ appears on the fraction analysis by SDS-PAGE corresponding to SpCas9 as the major protein. In the second step, size exclusion chromatography, there were three main peaks with an elution volume of 8.8,13.6,16.3 $\mathrm{ml}$ (B). The first peak $(8.8 \mathrm{ml})$ shows several bands containing the $160 \mathrm{kDa}$ protein, the samples were collected and also analyzed by SDSPAGE (Fig. 2A-B)The fraction corresponding to the second peak (13.6 ml) shows a band of $160 \mathrm{kDa}$. The third peak $(16.3 \mathrm{ml})$ shows a mixture of contaminated low molecular size proteins (Fig. 2B). There is also a fourth minor peak eluting at $20.6 \mathrm{ml}$, consistent with the conductivity peak corresponding to the imidazole (68 Da).

Figure 3. Characterization of the purified SpCas9. Our two-step purification method produced a high enzymatic activity SpCas9-6xHis. In the absence of sgRNA targeting the fluorochrome region of the GFP gene using a DNA substrate (PCR product), had no background nuclease activity. The RNP complex sgRNA and the DNA substrates were incubated and there was no difference in the efficiency of specific doublestrand nuclease activity of both gRNAs (96.2\% vs. 95.4\%).

Figure 4. Electroporation of GFP-expressing bacteria with the RNP. The transformation Cas9 and sgRNA by electroporation resulted in loss of plasmid-expressed GFP fluorescence in $E$. coli colonies. A short DNA recombination template (30 bp homology arm) encoding the mutation responsible for the transition from GFP to BFP 
(Tyr66 $\rightarrow$ His) was also used to turn blue in RNP-transformed cells. These results are proof of the principle of use of RNP in bacteria.

Figure 5. Cells with successful reparation produced fluorescence in color blue. Fluorescent cells can be observed after the electroporation(Fig. 5A). Although the observed efficiency was low $(<5 \%)$, we could see a proof of principle for the use of RNPs in bacteria. As the transformation efficiency of SpCas $9 /$ gRNA complex would expectedly be limited due to its massive size, as we can see in the section B. Recombineering efficiency must be maximized.

Figure S1. Gel filtration of molecular weight standards. Expected molecular size of several proteins on the Superdex 200 increase 10/300 GL column. Elution of SpCas9-6xhis (160 $\mathrm{kDa}$ ) at $13.6 \mathrm{ml}$ is consistent with its molecular weight according to this chromatogram, near to the elution time of aldolase, peak 3 with a MW of $158 \mathrm{kDa}$ 


\section{Figures}

Gene editing in bacteria via SpCas9/sgRNA ribonucleoprotein complexes 
Fig. 1

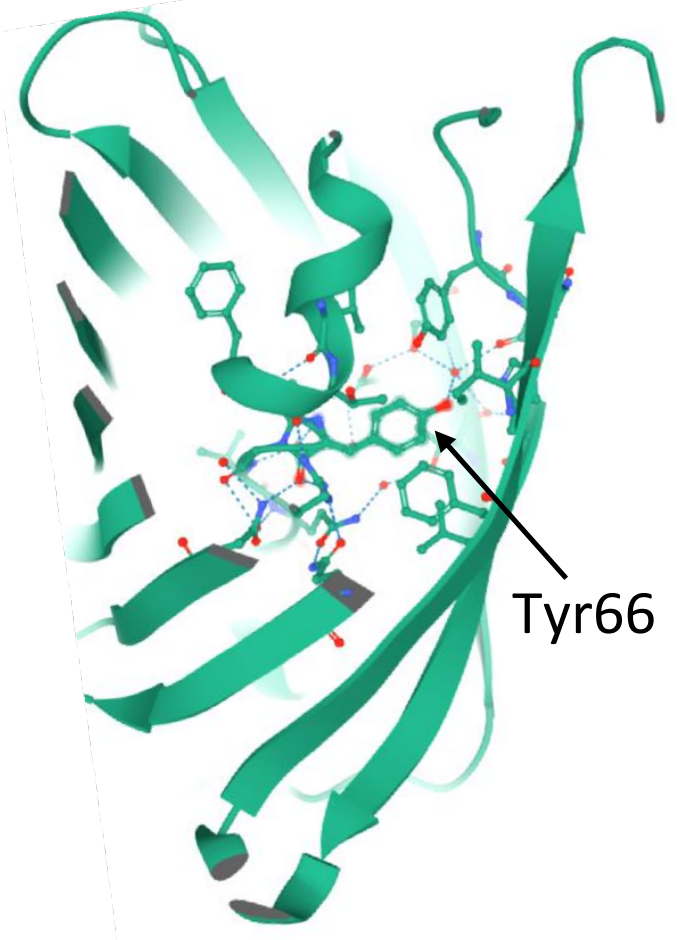

GFP

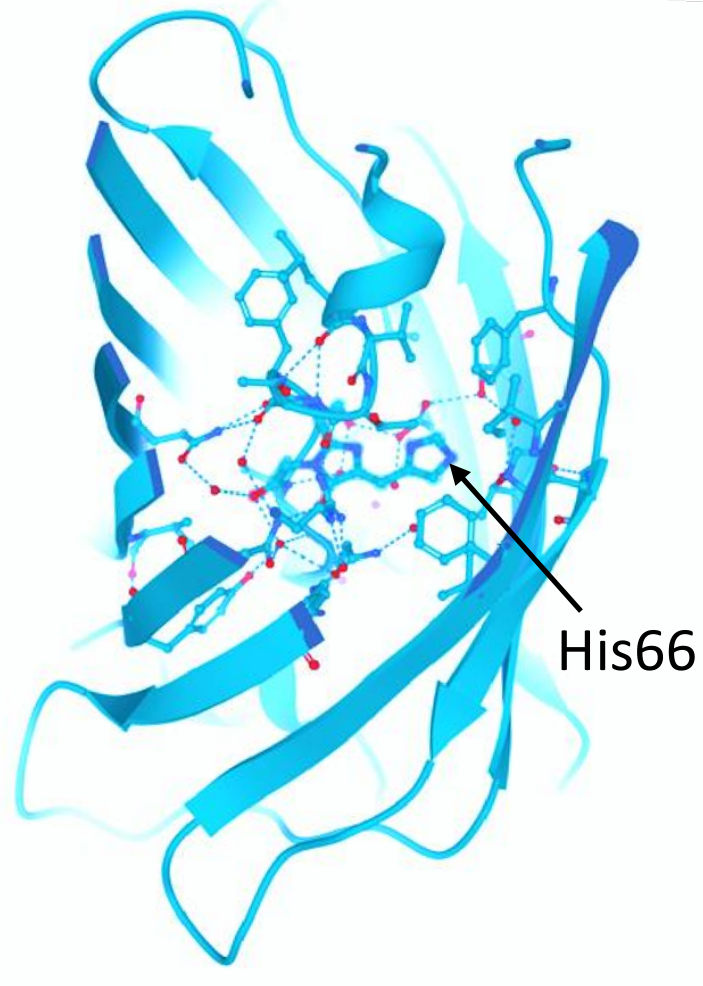

BFP
GFP

BFP cttgtcactactttctcttatggtgttcaatgctttgcgagataccca

$$
\begin{array}{l|l|l|l|l|l|l|l|l|l|l|l|l|l|l|l|}
\text { L } & \text { V } & \text { T } & \text { T } & \text { F } & \text { S } & Y & \text { G } & \text { V } & \text { Q } & \text { C } & \text { F } & \text { A } & \text { R } & \text { Y } & \text { P }
\end{array}
$$

cttgtcactactctgagccacggtgttcaatgctttgcgagataccca
L
T L
\begin{tabular}{l|l|l|l|l} 
& $H$ & $G$ & $V$ & 0
\end{tabular}
$\begin{array}{lllllll}C & F & A & R & Y & P\end{array}$
60
65
70
75 
Fig. 3

GFPmut

cttgtcactactttcgggtatggtgttcaatgctttgcgagatacccagatcatatgaaa $\begin{array}{lllllllllllllllllllll}\mathrm{L} & \mathrm{V} & \mathrm{T} & \mathrm{T} & \mathrm{F} & \mathrm{G} & \mathrm{Y} & \mathrm{G} & \mathrm{V} & \mathrm{Q} & \mathrm{C} & \mathrm{F} & \mathrm{A} & \mathrm{R} & \mathrm{Y} & \mathrm{P} & \mathrm{D} & \mathrm{H} & \mathrm{M} & \mathrm{K}\end{array}$

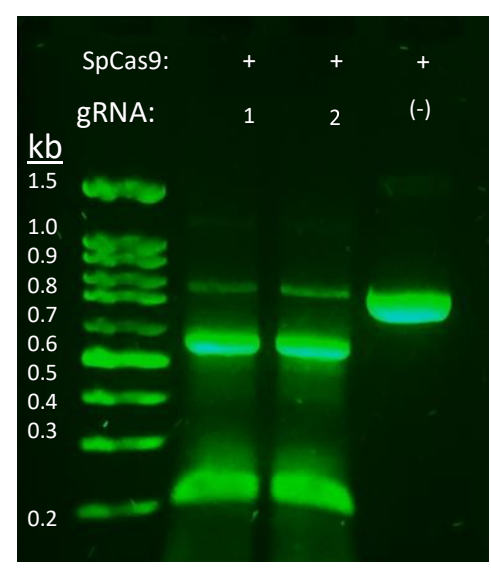

\author{
gRNA1: cttgtcactactttcggtta
}

gRNA2: cttgtcactactttcgggta

\begin{tabular}{l|c|c|c|} 
& gRNA1 & gRNA2 & No gRNA \\
\cline { 2 - 4 } Uncut (\%) & 3.8 & 4.6 & 100.0 \\
\hline Cut (\%) & 96.2 & 95.4 & 0.0
\end{tabular}


Fig. 4
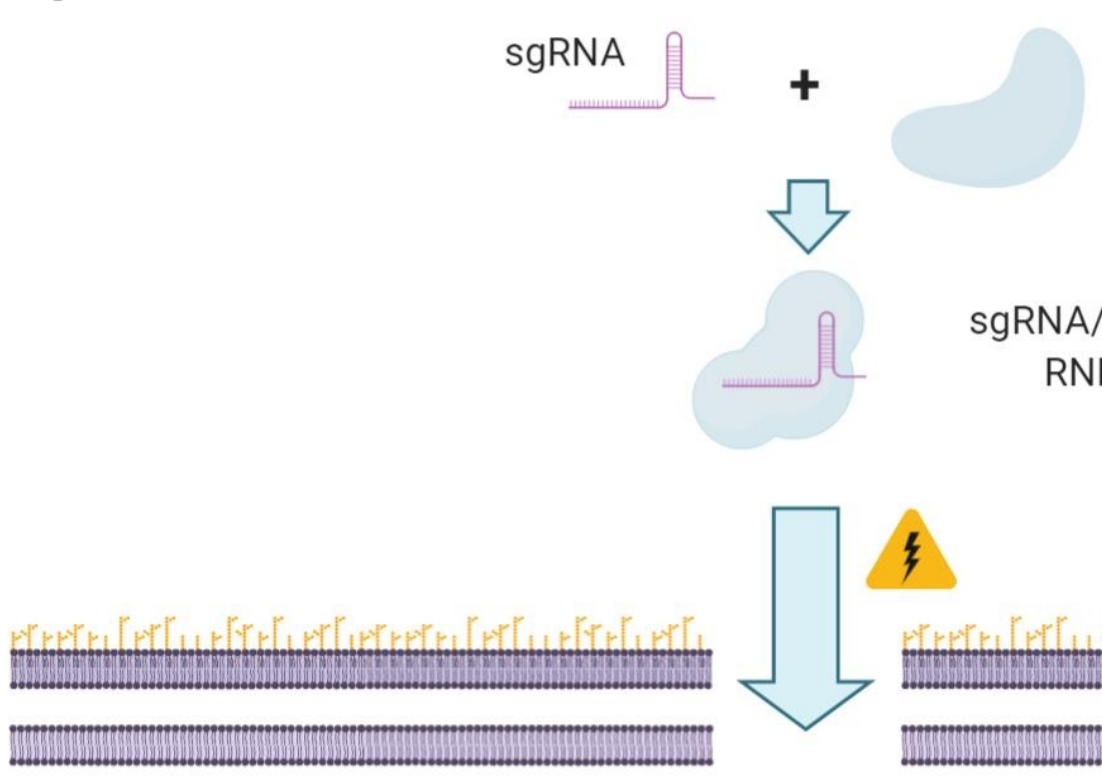

Cas9
sgRNA/Cas 9
RNP

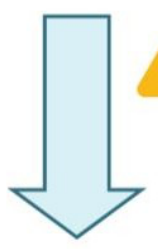

\section{s}
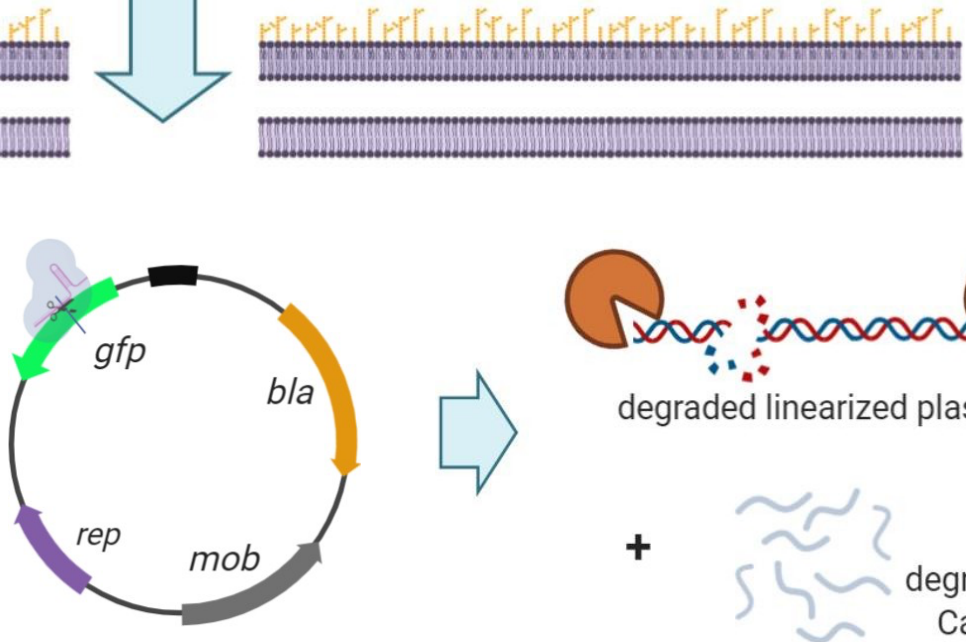

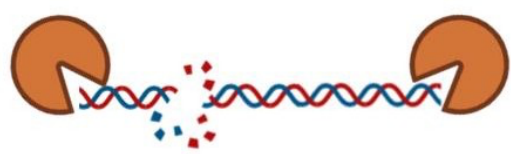

degraded linearized plasmid
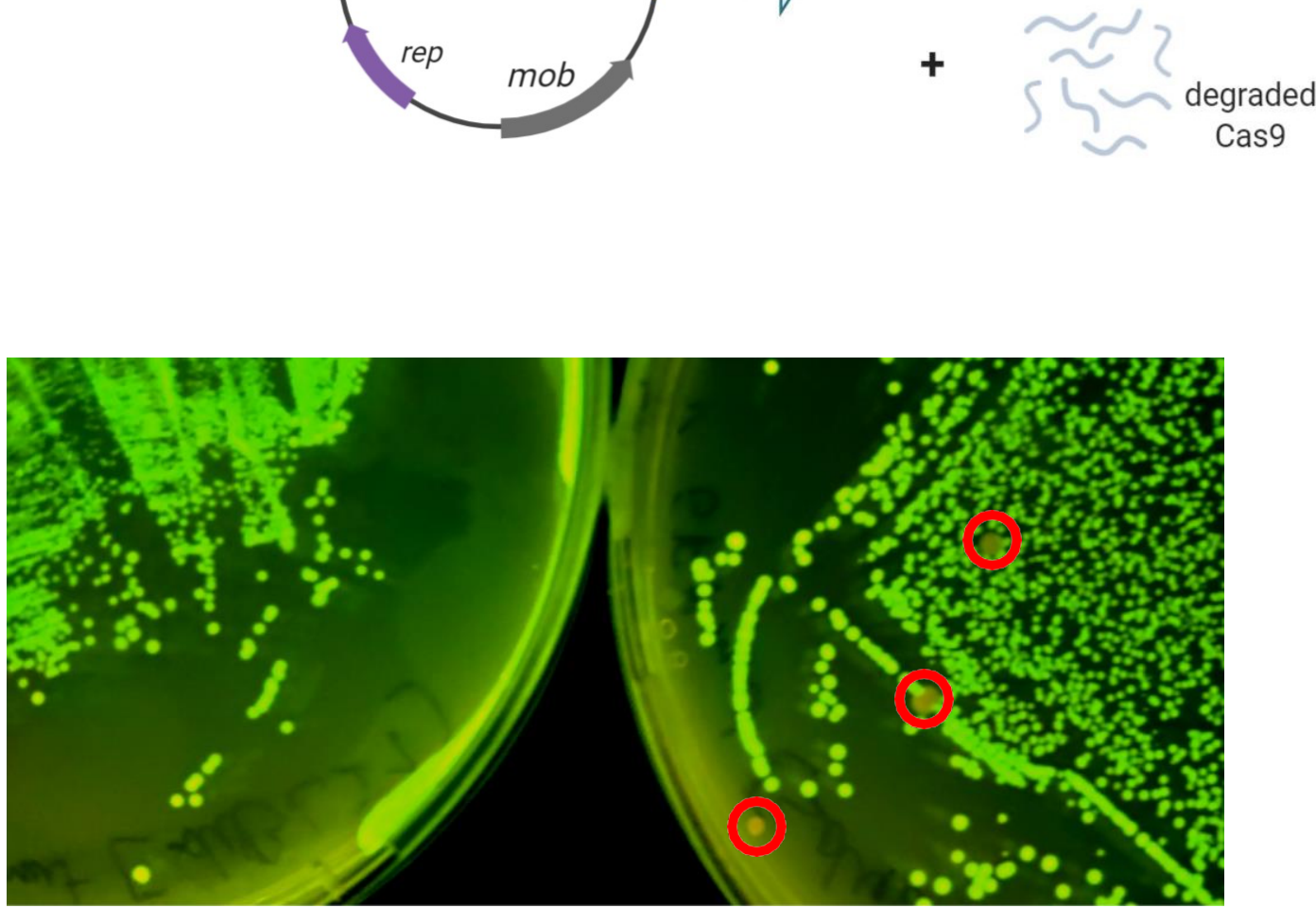


\section{Fig. 5}
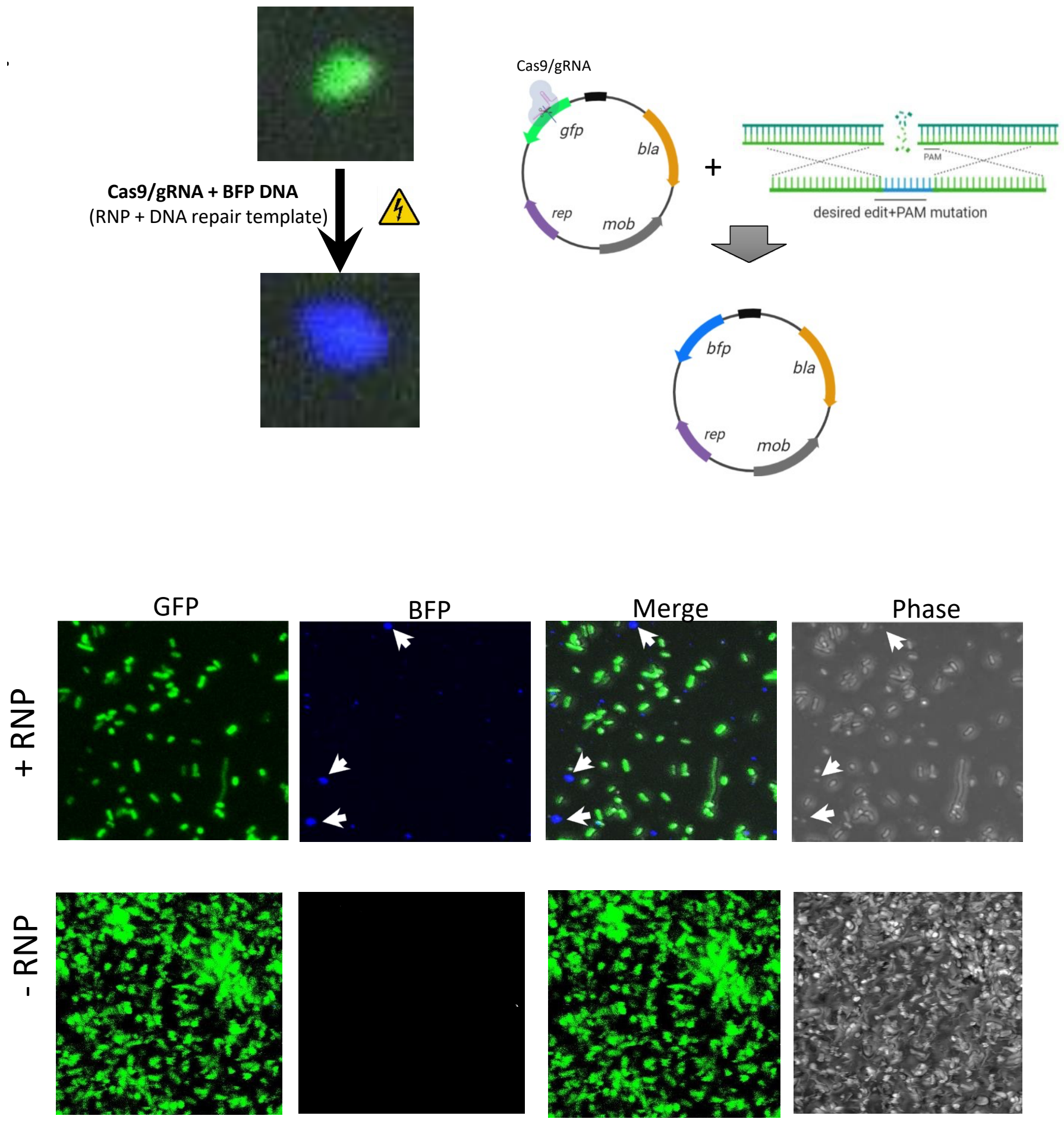
Fig. S1

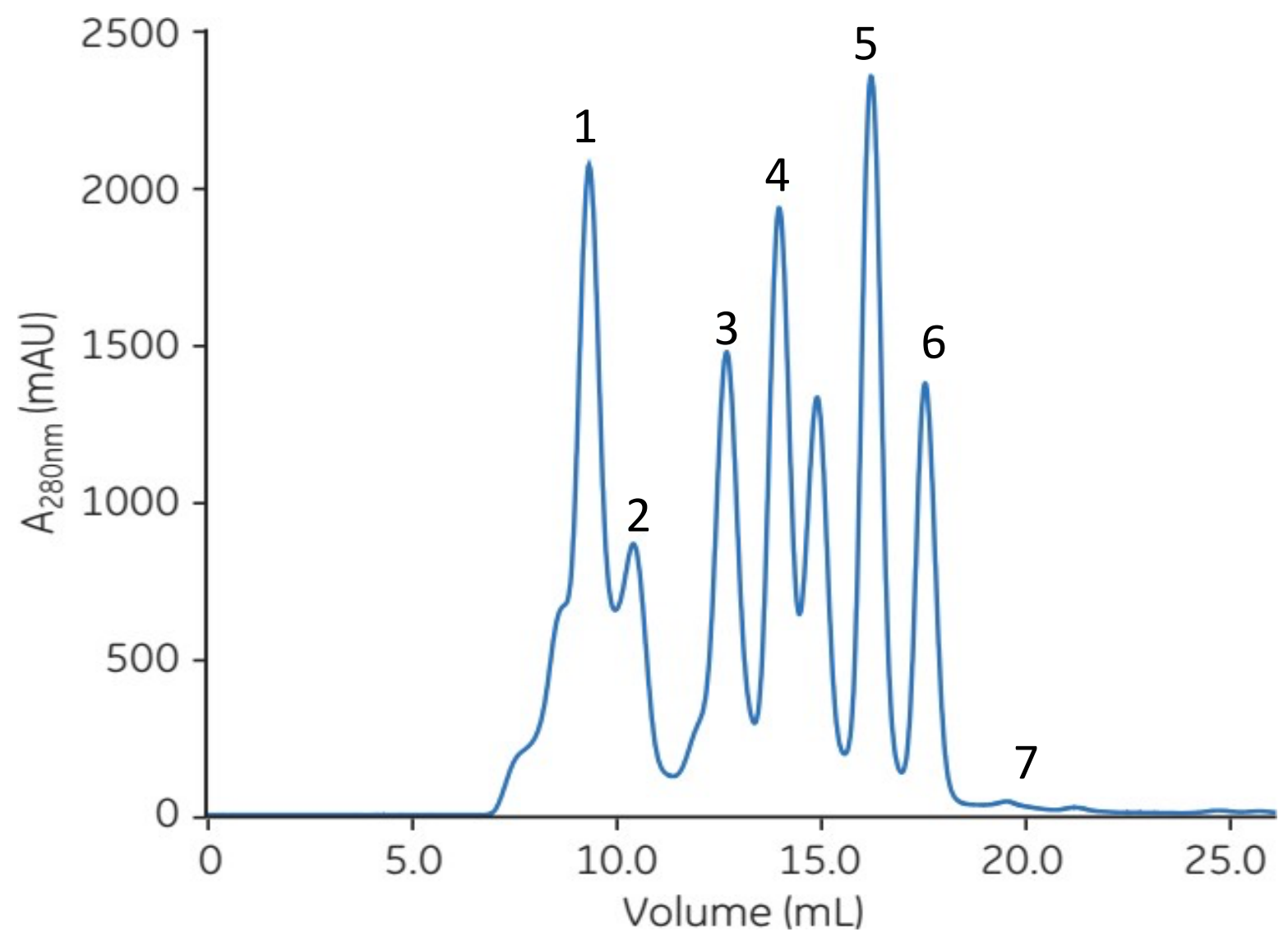

Column:

Superdex 200 Increase 10/300 GL

Sample:

1. Thyroglobulin (M, 669 000), $3 \mathrm{mg} / \mathrm{ml}$

2. Ferritin (M 440000$), 0.3 \mathrm{mg} / \mathrm{ml}$

3. Aldolase $\left(M_{r} 158000\right), 3 \mathrm{mg} / \mathrm{ml}$

4. Conalbumin (Mr 75000$), 3 \mathrm{mg} / \mathrm{ml}$

5. Ovalbumin (Mr 44000$), 3 \mathrm{mg} / \mathrm{ml}$

6. Carbonic anhydrase $(M, 29000), 3 \mathrm{mg} / \mathrm{ml}$

7. Ribonuclease A (M, 13 700), 3 mg/ml 